\title{
GNAO1 as a Novel Predictive Biomarker for Late Relapse in Hepatocellular Carcinoma
}

\author{
Meiling Du $\mathbb{D}^{1},{ }^{1}$ Jie Feng $\mathbb{D}^{1},{ }^{1}$ Yiran Tao $\mathbb{D}^{2},{ }^{2}$ Qincong Pan $\mathbb{D}^{1},{ }^{1}$ and Fengyuan Chen $\mathbb{D}^{1}$ \\ ${ }^{1}$ Department of Gastroenterology, Shanghai Fifth People's Hospital, Shanghai 201200, China \\ ${ }^{2}$ Department of General Medicine, Zhoupu Community Health Service Center, Shanghai 201100, China
}

Correspondence should be addressed to Fengyuan Chen; chenfengyuan_@fudan.edu.cn

Received 8 September 2021; Accepted 21 October 2021; Published 1 December 2021

Academic Editor: Nahrizul Adib Kadri

Copyright (c) 2021 Meiling Du et al. This is an open access article distributed under the Creative Commons Attribution License, which permits unrestricted use, distribution, and reproduction in any medium, provided the original work is properly cited.

GNAO1, the alpha $\mathrm{O} 1$ subunit of $G$ protein, was reported to be significantly downregulated in hepatocellular carcinoma (HCC), as well as being implicated in a variety of intracellular biological events; findings suggest that it may act as a tumor suppressor. Our goal was to further explore the expression of GNAO1 in HCC patients and its potential clinical significance. Oncomine and Kaplan-Meier plotter databases were used to assess the mRNA expression of GNAO1 in HCC tissues and patient survival time. Subsequently, immunohistochemistry (IHC) was used to measure GNAO1 protein level in tissue from 79 cases of HCC and paired adjacent tissues. The Kaplan-Meier survival analysis, Cox regression model, and prognostic nomogram were used to evaluate the prognostic role of GNAO1 in HCC. Results demonstrated that mRNA and protein expressions of GNAO1 were both lower in HCC tissues than in adjacent tissues (all $p<0.01$ ). HCC patients with high expression of GNAO1 had better relapse-free survival (RFS) than those with low GNAO1 expression (all $p<0.05$ ). A high expression of GNAO1, meanwhile, functioned as a good predictor of late relapse for HCC $(p<0.05)$. The nomogram consisting of GNAO1 expression and the tumor-node-metastasis (TNM) model presented good ability in predicting the 3-year relapse for HCC (C-index $=0.614)$. In conclusion, GNAO1 was a reliable biomarker of relapse prediction for HCC.

\section{Introduction}

Liver cancer is one of the most lethal and prevalent cancers worldwide and has recently become the third leading cause of cancer-related mortality in humans [1]. Hepatocellular carcinoma (HCC) is the primary cancer of the liver, accounting for almost $75 \%$ of all liver cancers globally [2]. Established major risk factors for HCC include chronic infection with hepatitis B virus (HBV) and hepatitis $C$ virus, excess alcohol consumption, and nonalcoholic fatty liver disease. Despite of recent progress in diagnosis and treatment, the clinical outcomes of HCC patients remain very poor. Thus, it is particularly crucial to explore a novel biomarker of early diagnosis or prognostication for HCC.

GNAO1 encodes the alpha O1 subunit of guanine nucleotide-binding proteins, which can trigger signaling cascades and regulate several cellular events when they bind to a serpentine receptor [3]. GNAO1, first mentioned in 1987
[4], is located on chromosome $16 \mathrm{q} 13$ and is predominantly localized in nervous tissues and neuroendocrine cells [5]. Previous research focused on GNAO1 mutation-induced encephalopathy, including hypotonia, epilepsy, and dyskinesia [6-9]. Additionally, GNAO1 dysfunction or aberrant expression could promote oncogenesis such as in gastric cancer [10], colorectal cancer [11, 12], and breast cancer [13].

As an association with HCC, Pei et al. found that GNAO1 was downregulated in HCC cells and that the silencing of GNAO1 by small-interfering RNA increased the proliferation, while inhibiting the senescence of HCC cells [14]. Lately, researchers, including our own team, further elucidated that low expression of GNAO1 in HCC cells was partly attributed to DNA methyltransferase 1 and the deacetylase silent information regulator 1 indirectly mediating the promoter hypermethylation $[15,16]$. In clinical study, Pei et al. also demonstrated that GNAO1 was comparably less in HCC tissues than in adjacent tissues by 
immunohistochemistry (IHC) on 20 paired samples [14]. However, very small sample size and absence of a statistical test in the study are incomplete for us to understand the expression of GNAO1 in HCC patients. Moreover, the potential clinical significance of GNAO1 remains unknown.

In our study, bioinformatics data were studied, with the IHC assay aiming to define the trait and the role of GNAO1 in HCC patients, based on the effective statistical analysis. We hope to provide a good prognostic biomarker and a new target for anticancer therapy in HCC.

\section{Materials and Methods}

2.1. Oncomine Database Analysis. The differential expression of GNAO1 mRNA between HCC and normal liver tissues was reviewed in the Oncomine database (https:// www.oncomine.org/resource/login.html), which is a cancer microarray database and web-based data-mining platform aimed at facilitating discovery from genome-wide expression analysis [17]. The threshold settings were as follows: gene ranking of top $10 \%$, a fold change of 2.0 , and a $p$ value of $1 \mathrm{E}-4$.

\subsection{Kaplan-Meier Plotter Database Analysis. The} Kaplan-Meier plotter database (http://www.kmplot.com), an online survival analysis tool, was used to evaluate relationships between gene expression and patient prognosis, such as overall survival (OS) and relapse-free survival (RFS), across a large collection of publicly available cancer microarray datasets [18]. Patient samples were split into two groups according to the best cutoff of GNAO1 expression (low versus high expression) and were assessed by a Kaplan-Meier survival plot, with the hazard ratio (HR) with 95\% confidence interval (CI) and $\log \operatorname{rank} p$ value.

2.3. Clinical Samples. A human liver cancer tissue microarray (cat no. LVC1605) containing paired tumor and normal tissue from 80 cases, together with patient clinical data, was purchased from Shanghai zhuolibiotech Co., Ltd. (Shanghai, China). All patients received radical surgery from January 2004 to June 2012. Patients who were pathologically diagnosed with primary HCC were adopted into the study. Exclusion criteria were a history of radiotherapy or chemotherapy before surgery and less than 36 months of followup.

2.4. IHC Assay. According to the instructions of the UltraSensitive SP detection kit (KIT-9730, Maxim Biotech, Fuzhou, China), IHC staining was performed on HCC tissue microarray to detect the expression of GNAO1 protein. Briefly, tissue sections were deparaffinized with xylene and rehydrated, followed by microwave antigen retrieval. After being blocked with hydrogen peroxidase and nonimmune animal serum, the sections were incubated at $4^{\circ} \mathrm{C}$ overnight with the primary antibody against GANO1 (1 :200, \#126351-AP, Proteintect Group, Rosemont, USA). The sections were further incubated in the solution of biotin-labeled secondary antibody at room temperature for 30 minutes and then in streptavidin peroxidase reagent for 10 minutes. The sections should be rinsed in PBS thrice after each step (each rinse for five minutes). Finally, IHC staining was conducted using a DAB kit (P0203, Beyotime Biotech, Shanghai, China).

2.5. Semiquantitative Analysis. The immunohistochemical staining result was evaluated using a semiquantitative method (immunoreactive score, IRS) as described previously [19]. IRS (0-12) for each slice was calculated by multiplying the staining intensity in four gradations ( 0 , negative; 1, weak; 2 , moderate; and 3 , strong) with the percentage of positive cells in five gradations ( 0 , negative; 1 , $\leq 10 \% ; 2,11-50 \% ; 3,51-80 \%$; and $4, \geq 81 \%$ ), and each specimen was measured in three different magnification fields. Two pathologists independently observed the staining results under double-blind conditions.

2.6. Statistical Analysis. Statistical analysis was made in SPSS (version 22.0) software and $R$ (version 4.1.0.) software. The measurement data with normal distribution were expressed as means \pm standard deviations, and nonnormally distributed data were expressed as the median. We performed Student's $t$-test and Pearson's chi-squared test to compare the difference between groups. Moreover, Kaplan-Meier curves were drawn to compare the prognostic difference between groups. The prognostic performance of factors was explored via univariable and multivariable Cox proportional hazard regression analysis. Then, the predictive performance of factors was assessed via time-dependent receiver operating characteristic (ROC) curves and the area under the curve (AUC) by using the "survival ROC" package of $R$ software. Nomogram, a powerful tool for quantifying each prognostic factor on the survival [20], was created based on the Cox regression model by using the "rms" package. The calibration curve and C-index were generated to analyze the agreement between the nomogram and ideal observation. $p$ value less than 0.05 was considered statistically significant.

\section{Results}

3.1. Patient Characteristics. A total of 79 cases of primary HCC were adopted into the group, with the median followup of 42 months (range: 5-84 months). The median age of patients at diagnosis was 54 years, and $17(21.5 \%)$ patients were aged 60 years and older. Sixty-six patients $(83.5 \%)$ were male, and $13(16.5 \%)$ were female. The number of patients with tumor stage I, II, III, and IV was 30 (38.0\%), 34 (43.0\%), $3(3.8 \%)$, and $12(15.2 \%)$, respectively. Detailed clinical parameters of 79 HCC patients are shown in Table 1.

\subsection{Low mRNA and Protein Levels of GNAO1 in HCC Tissues.} There were 5 RNA chips with a total of 413 HCC and 340 normal liver tissues involved in the analysis of differential expression of GNAO1 mRNA in the Oncomine database. Results showed that the mRNA expression of GNAO1 was 
TABLE 1: Relationships between GNAO1 protein level and clinicopathological parameters in 79 HCC patients.

\begin{tabular}{|c|c|c|c|c|}
\hline \multirow{2}{*}{ Clinicopathological parameters } & \multirow{2}{*}{ Case, $n(\%)$} & \multicolumn{2}{|c|}{ GNAO1 expression } & \multirow{2}{*}{$p$ value } \\
\hline & & Low $(n=35)$ & High $(n=44)$ & \\
\hline \multicolumn{5}{|l|}{ Age (years) } \\
\hline$<60$ & $62(78.5)$ & 29 & 33 & 0.399 \\
\hline$\geq 60$ & $17(21.5)$ & 6 & 11 & \\
\hline \multicolumn{5}{|l|}{ Sex } \\
\hline Male & $66(83.5)$ & 29 & 37 & 0.883 \\
\hline Female & $13(16.5)$ & 6 & 7 & \\
\hline \multicolumn{5}{|l|}{ HBV infection } \\
\hline Yes & $62(78.5)$ & 26 & 36 & 0.418 \\
\hline No & $17(21.5)$ & 9 & 8 & \\
\hline \multicolumn{5}{|l|}{$\mathrm{AFP}$} \\
\hline$<200 \mathrm{ng} / \mathrm{mL}$ & $30(38.0)$ & 16 & 14 & 0.206 \\
\hline$\geq 200 \mathrm{ng} / \mathrm{mL}$ & $49(62.0)$ & 19 & 30 & \\
\hline \multicolumn{5}{|l|}{ Liver cirrhosis } \\
\hline Yes & $55(69.6)$ & 26 & 29 & 0.421 \\
\hline No & $24(30.4)$ & 9 & 15 & \\
\hline \multicolumn{5}{|l|}{ Tumor location } \\
\hline Left lobe & $42(53.2)$ & 16 & 26 & 0.237 \\
\hline Right lobe & $37(46.8)$ & 19 & 18 & \\
\hline \multicolumn{5}{|l|}{ Maximum tumor size } \\
\hline$<30 \mathrm{~mm}$ & $13(16.5)$ & 8 & 5 & 0.171 \\
\hline$\geq 30 \mathrm{~mm}$ & $66(83.5)$ & 27 & 39 & \\
\hline \multicolumn{5}{|l|}{ Number of tumors } \\
\hline 1 & $72(91.1)$ & 32 & 40 & 0.751 \\
\hline$\geq 2$ & $7(8.9)$ & 3 & 4 & \\
\hline \multicolumn{5}{|l|}{ Tumor type } \\
\hline Lump & $71(89.9)$ & 30 & 41 & 0.473 \\
\hline Nodular & $8(10.1)$ & 5 & 3 & \\
\hline \multicolumn{5}{|l|}{ Vascular invasion } \\
\hline Yes & $26(32.9)$ & 11 & 15 & 0.802 \\
\hline No & $53(67.1)$ & 24 & 29 & \\
\hline \multicolumn{5}{|l|}{ TNM stage (AJCC 8th) } \\
\hline I-II & $64(81.0)$ & 31 & 33 & 0.215 \\
\hline III-IV & $15(19.0)$ & 4 & 11 & \\
\hline
\end{tabular}

Abbreviations: HCC: hepatocellular carcinoma, HBV: hepatitis B virus, AFP: alpha-fetoprotein, TNM: tumor-node-metastasis, AJCC: American Joint Committee on Cancer

significantly lower in HCC tissues than in normal liver tissues (Figure 1).

To confirm the predictive results, IHC assay on 79 paired HCC samples was performed to examine GNAO1 protein level. The mean \pm standard deviation IRS of GNAO1 in cancerous tissues and noncancerous tissues was $6.43 \pm 3.93$ and $10.95 \pm 2.25$, respectively. The IRS of GNAO1 in the tumor was much lower than that in adjacent tissues $(t=9.840, p<0.001$; Figure 2$)$, indicating that the protein expression of GNAO1 was significantly lower in HCC tissues than in adjacent tissues. Results also showed that GNAO1 protein was mainly distributed on the membrane and in the cytoplasm of liver cells.

3.3. Relationships between GNAO1 Expression and Patient Clinicopathological Parameters. In our study, the median IRS scores of GNAO1 in 79 HCC tissues was 8. IRS ranged from 0 to 12 , with IRS $\geq 8$ indicating high GNAO1 expression and IRS $<8$ indicating low GNAO1 expression.
There were 44 patients with high expression of GNAO1 and 35 patients with low expression of GNAO1. As shown in Table 1, the differential expression of GNAO1 protein (low versus high) was not significantly associated with patient clinical characteristics, including age, sex, HBV infection, alpha-fetoprotein values, and liver cirrhosis, as well as pathological data, including tumor location, tumor size, tumor number, tumor type, vascular invasion, and tumornode-metastasis (TNM) stage.

3.4. High GNAO1 Expression Was Associated with Favorable RFS in HCC. In the Kaplan-Meier plotter database, the RFS analysis of all HCC patients $(n=316)$, stage I-II $(n=228)$, and stage III-IV $(n=70)$ revealed that patients with high mRNA expression of GNAO1 had more favorable RFS than those with low GNAO1 expression (all $p<0.05$; Figures 3(a)-3(c)).

We also analyzed the IHC staining results and found that RFS of HCC patients with high expression of GNAO1 

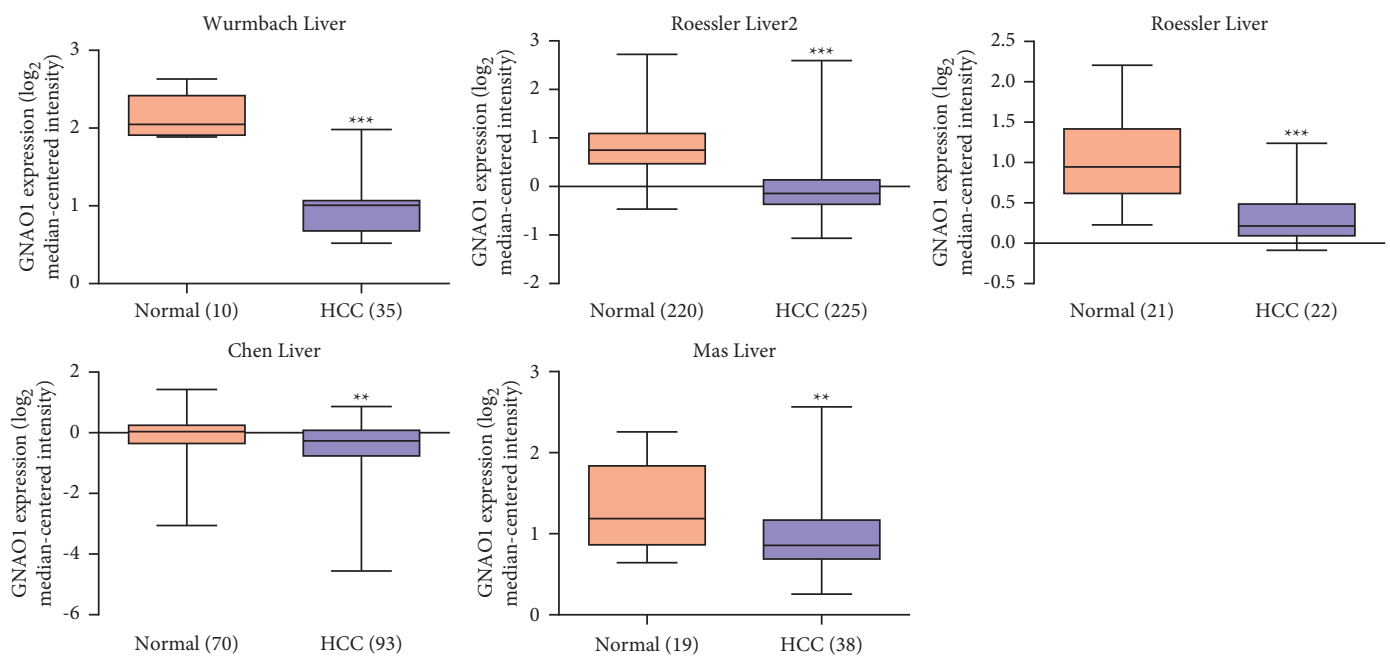

Figure 1: Expression of GNAO1 mRNA was lower in HCC tissues than in normal liver tissues based on the Oncomine data. Research samples were Wurmbach Liver $(t=10.249, p=4.069 E-13)$, Roessler Liver $2(t=21.263, p=1.440 E-66)$, Roessler Liver $(t=5.363$, $p=6.000 E-6)$, Chen Liver $(t=3.164, p=0.002)$, and Mas Liver $(t=3.409, p=0.002)$, respectively. ${ }^{* * *} p<0.001,{ }^{* *} p<0.01$.
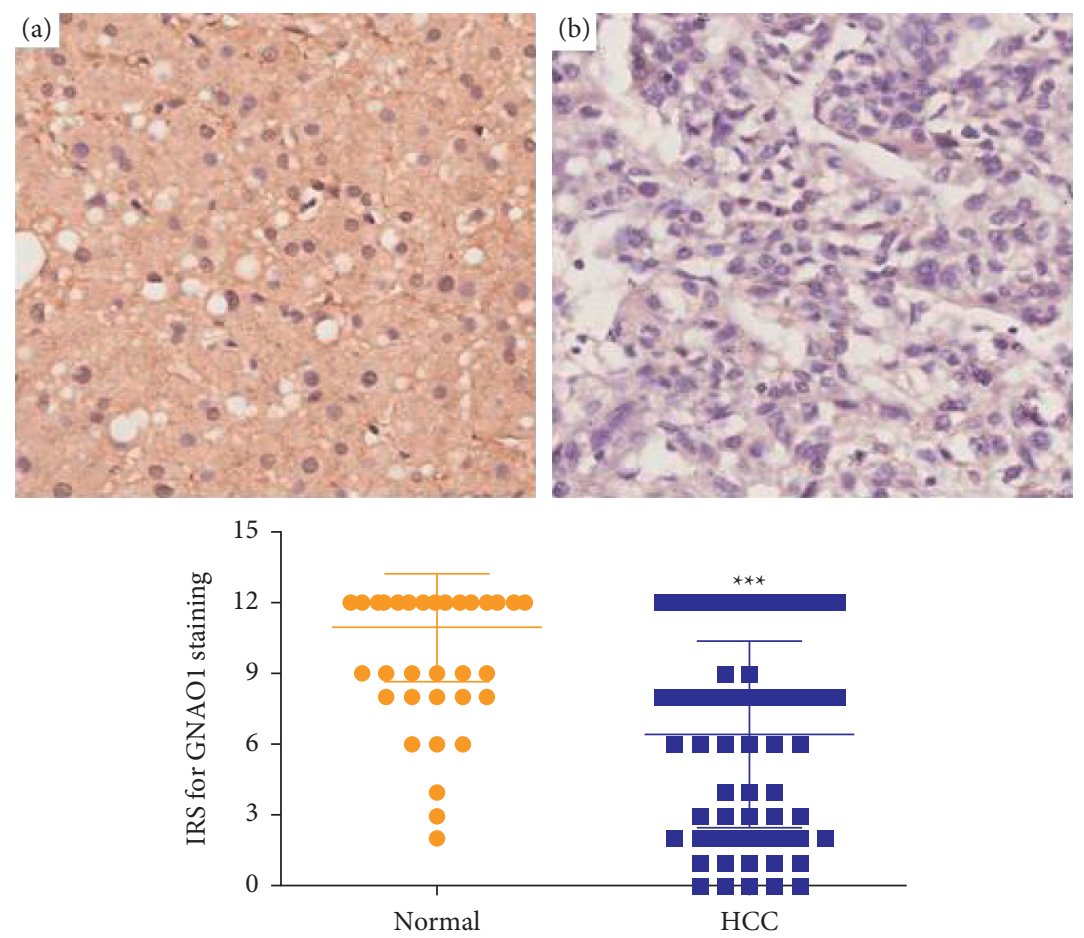

(c)

Figure 2: Expression of GNAO1 protein was lower in HCC tissues than in adjacent normal tissues based on the IHC results. (a) Representative high expression of GNAO1 in adjacent tissue, magnification: $\times 400$; (b) representative low expression of GNAO1 in HCC tissue, magnification: $\times 400$; and (c) the IRS in HCC tissues was statistically lower than that in adjacent normal tissues $(t=9.840, p=2.567 E-15) .{ }^{* * *} p<0.001$.

protein was significantly better than those with low GNAO1 expression (all $p<0.05$; Figures $3(\mathrm{~d})-3(\mathrm{e})$ ). The average RFS in the high GNAO1 expression cohort was longer than that in the low GNAO1 expression cohort $(31.36 \pm 22.97$ vs. $20.31 \pm 13.38$ months, $p<0.01)$.
3.5. GNAO1 Was a Reliable Biomarker for Relapse Prediction in HCC. Both univariate and multivariate Cox regression analysis indicated that GNAO1 expression was a negative factor significantly associated with HCC relapse $(\mathrm{HR}=0.512$, $95 \% \quad \mathrm{CI}=0.297-0.882, \quad p<0.05 ; \quad \mathrm{HR}=0.420, \quad 95 \%$ 


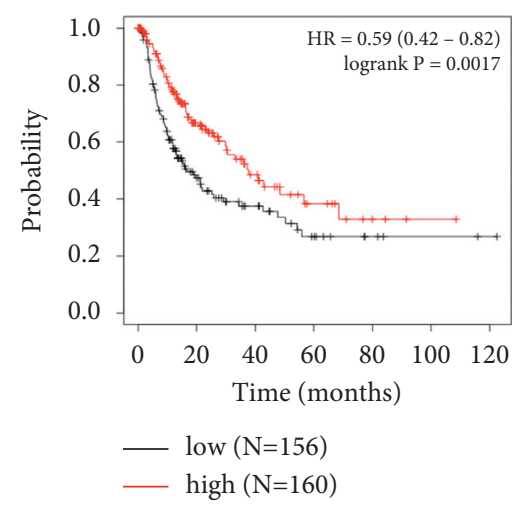

(a)

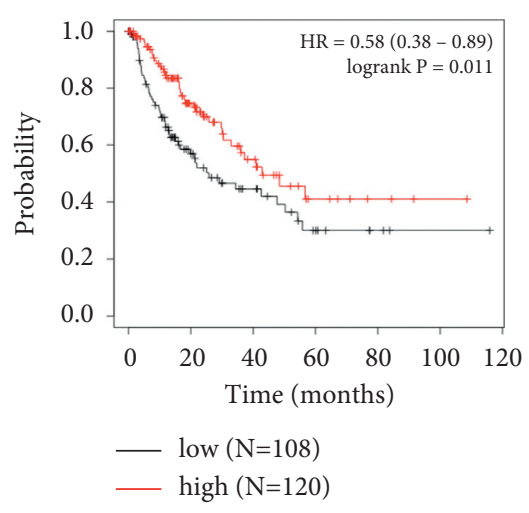

(b)

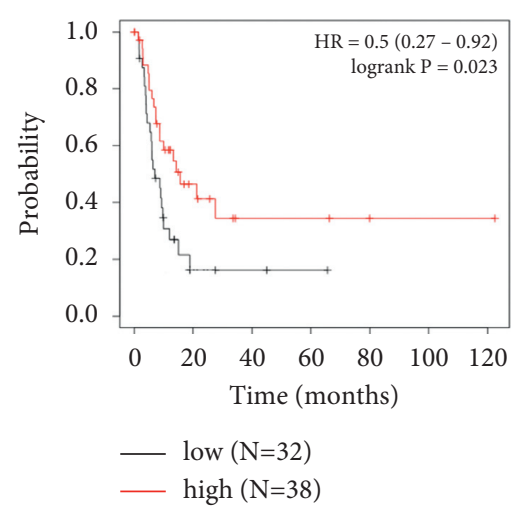

(c)

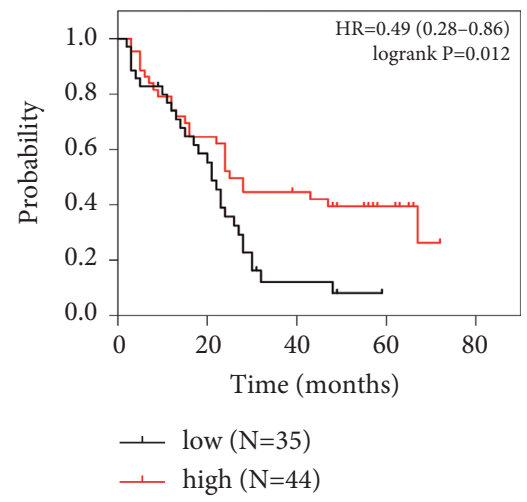

(d)

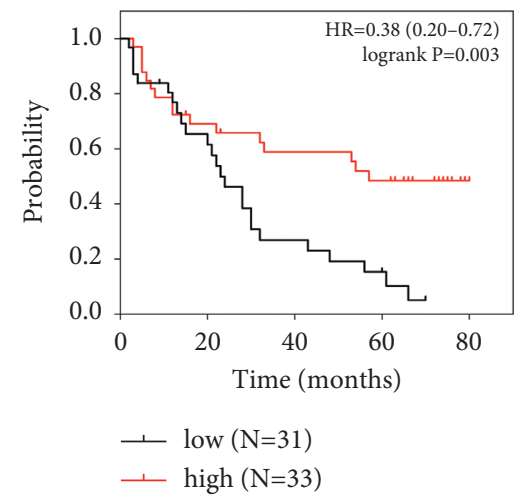

(e)

FIGURE 3: The Kaplan-Meier curves of RFS of HCC patients based on GNAO1 expression (low versus high). (a-c) RFS analysis of all HCC patients $(n=316)$, stages I-II $(n=228)$, and stages III-IV $(n=70)$ in the Kaplan-Meier plotter database; (d-e) RFS analysis of all HCC patients $(n=79)$ and stages I-II $(n=64)$ based on the IHC results.

$\mathrm{CI}=0.237-0.744, p<0.01$, respectively; Table 2). Subsequently, ROC analysis was conducted to assess how GNAO1 expression could behave in predicting relapse. As shown in Figure 4(a), the AUC of GNAO1 expression performed on relapse was 0.688 , which was superior to that of TNM stage (0.606) and other clinicopathological parameters.

To predict the probability of 1-, 2-, and 3-year relapse, a nomogram model was established (Figure 4(b)). Calculating the score of GNAO1 expression and TNM stage, a straight line was generated to evaluate the relapse probability at each time point. The calibration curve presented that the rate of predicted relapse in 3-year closely paralleled the actually observed ratio (C-index $=0.614$; Figure $4(\mathrm{c})$ ), indicating the agreement between model prediction and reality.

\section{Discussion}

Most information about GNAO1 in HCC stems from in vitro experiments and is, as yet, insufficient to really understand the function of GNAO1. In our study, we analyzed GNAO1 expression and its clinical significance in HCC patients by using bioinformatics data, IHC assay, and effective statistical analysis.

A total of 5 RNA chip analysis, involving 413 HCC and 340 normal liver samples, all revealed that GNAO1 mRNA expression was at a lower level in HCC tissues than in normal liver tissues. Meanwhile, GNAO1 protein expression was confirmed to be significantly downregulated in HCC tissues by IHC on 79 paired samples, concurring with the bioinformatics results and the report of Pei et al. [14].

Subsequently, a survival analysis was conducted using data from the Kaplan-Meier plotter database along with IHC results. The analysis showed that HCC patients with high GNAO1 expression had better RFS compared to those with low GNAO1 expression, regardless of whether the tumor was at an early or advanced stage. This result may be attributed to the biological function of GNAO1 in HCC cells, which inhibits proliferation and promotes apoptosis and senescence [14-16]. Notably, the range of RFS from IHC results was shorter than that in the database, which may be related to the short postoperative follow-up time of our patients. Besides, it is interesting that HCC patients with high GNAO1 expression did not have prolonged OS (Figure 5). It is well known that OS is not only affected by tumor relapse but is also subject to other factors, such as how the patient responds to retreatment [21].

In general, TNM stage is a systematic anatomic-based classification that provides a method to estimate cancer prognosis. It is believed that early-stage patients have better outcomes after surgical resection [22, 23]. In our study, TNM stage was not closely related to GNAO1 expression. However, the Cox regression model and ROC analysis 
TABLE 2: Cox survival analysis of GNAO1 protein level and clinicopathological parameters in 79 HCC patients

\begin{tabular}{|c|c|c|c|c|}
\hline \multirow{2}{*}{ Parameters } & \multicolumn{2}{|c|}{ Univariate } & \multicolumn{2}{|c|}{ Multivariate } \\
\hline & $\operatorname{HR}(95 \% \mathrm{CI})$ & $p$ value & $\operatorname{HR}(95 \% \mathrm{CI})$ & $p$ value \\
\hline GNAO1 level (low vs. high) & $0.512(0.297-0.882)$ & $0.016^{*}$ & $0.420(0.237-0.744)$ & $0.003^{* *}$ \\
\hline Age (years) $(<60$ vs. $\geq 60)$ & $0.947(0.498-1.800)$ & 0.868 & & \\
\hline Sex (male vs. female) & $1.442(0.680-3.057)$ & 0.340 & & \\
\hline HBV infection (yes vs. no) & $0.821(0.445-1.514)$ & 0.527 & & \\
\hline $\operatorname{AFP}(\mathrm{ng} / \mathrm{mL})(<200$ vs. $\geq 200)$ & $0.672(0.392-1.153)$ & 0.149 & & \\
\hline Liver cirrhosis (yes vs. no) & $0.841(0.474-1.492)$ & 0.553 & & \\
\hline Tumor location (left vs. right) & $1.101(0.644-1.881)$ & 0.725 & & \\
\hline Maximum tumor size $(\mathrm{mm})(<30$ vs. $\geq 30)$ & $1.156(0.545-2.452)$ & 0.706 & & \\
\hline Number of tumors ( 1 vs. $\geq 2)$ & $0.853(0.340-2.144)$ & 0.736 & & \\
\hline Tumor type (lump vs. nodular) & $0.590(0.213-1.683)$ & 0.311 & & \\
\hline Vascular invasion (yes vs. no) & $1.245(0.718-2.157)$ & 0.436 & & \\
\hline TNM stage (I-II vs. III-VI) & $1.959(1.055-3.638)$ & $0.033^{*}$ & $2.581(1.345-4.954)$ & $0.004^{* *}$ \\
\hline
\end{tabular}

Abbreviations: HCC: hepatocellular carcinoma, HR: hazard ratio, CI: confidence interval, HBV: hepatitis B virus, AFP: alpha-fetoprotein, TNM: tumor-nodemetastasis. ${ }^{* *} P<0.01,{ }^{*} P<0.05$.

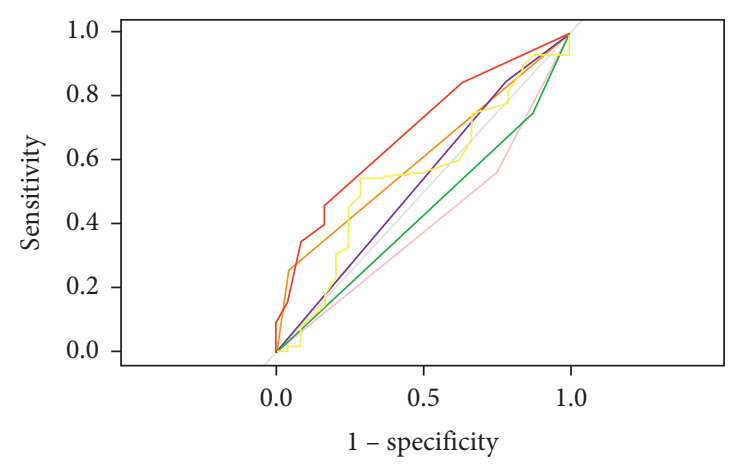

$$
\begin{aligned}
& -\operatorname{AUC}(\mathrm{GNAO} 1)=0.688-\operatorname{AUC}(\text { size })=0.531 \\
& \text { AUC }(\text { sex })=0.531-\operatorname{AUC}(\mathrm{TNM})=0.606 \\
& \text { AUC }(\text { age })=0.551-\operatorname{AUC}(\mathrm{AFP})=0.407 \\
& \text { AUC }(\mathrm{HBV})=0.435
\end{aligned}
$$

(a)

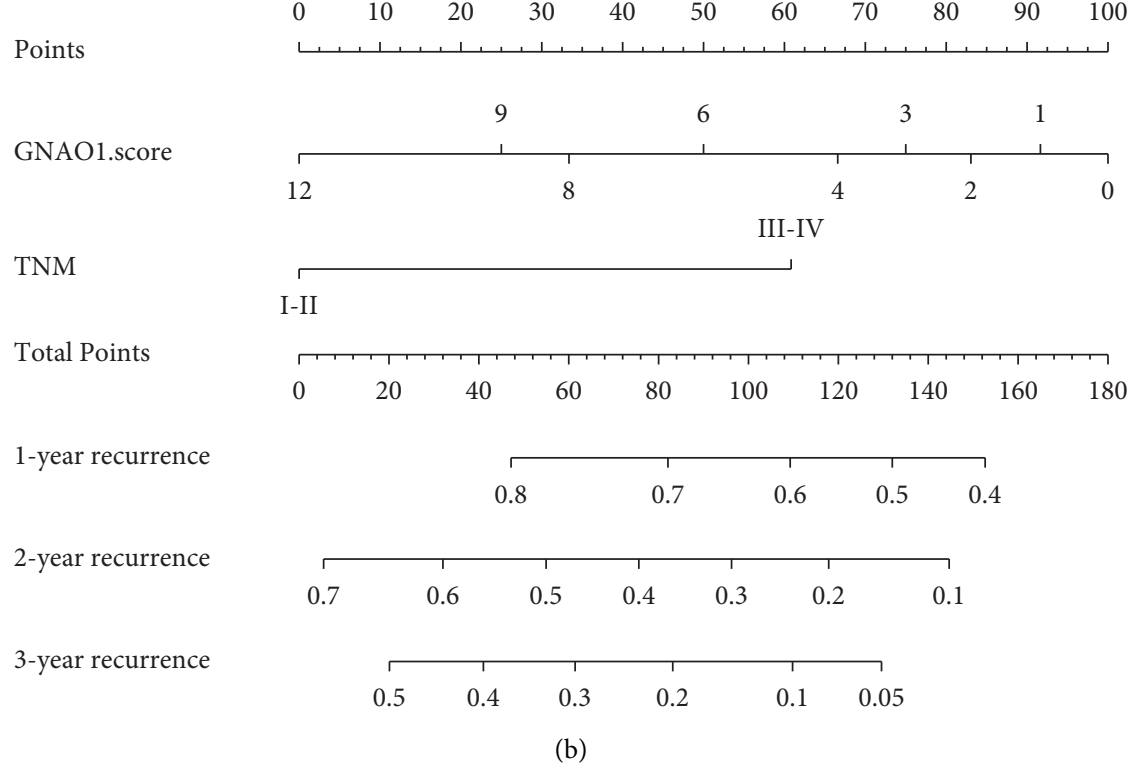

Figure 4: Continued. 


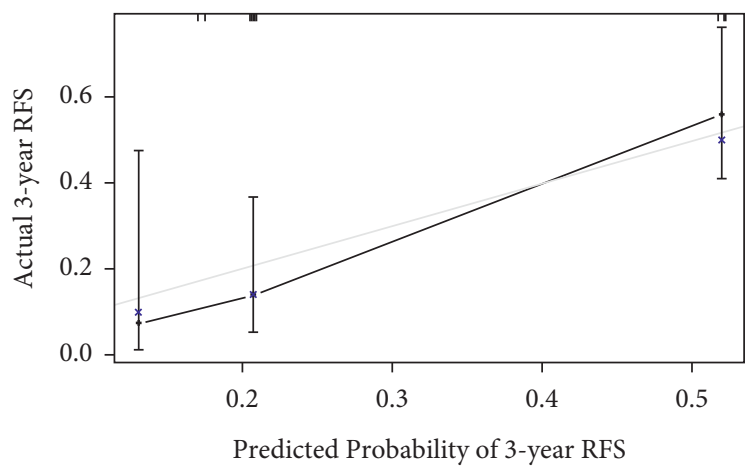

(c)

FIGURE 4: Construction of a prognostic model for HCC relapse assessment. (a) ROC analysis for GNAO1 expression and clinicopathologic parameters; (b) prognostic nomogram for HCC patients; and (c) calibration curves for the nomogram at 3 years.

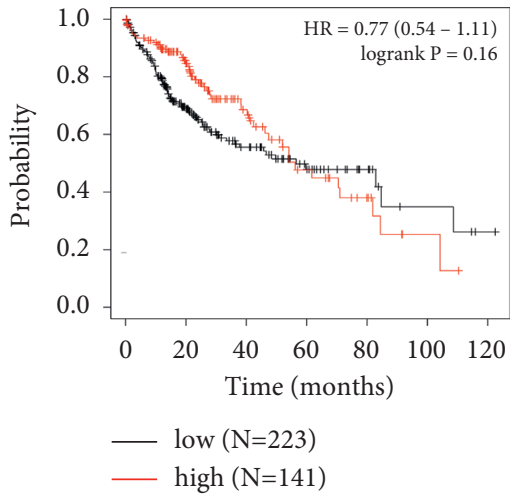

(a)

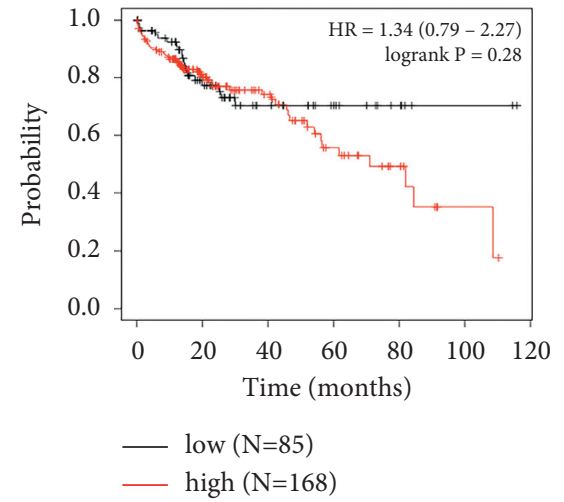

(b)

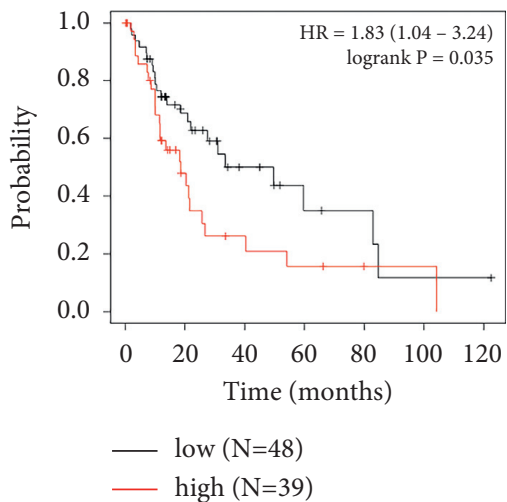

(c)

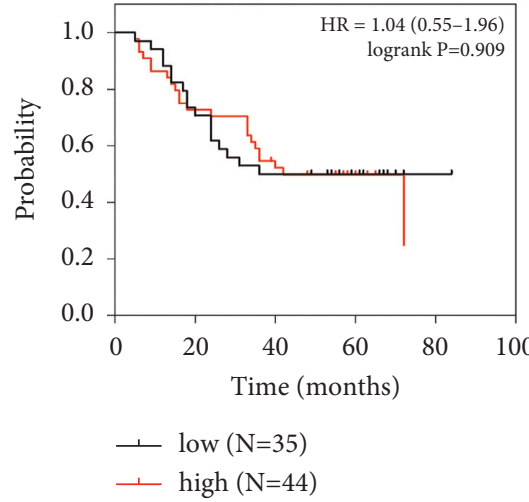

(d)

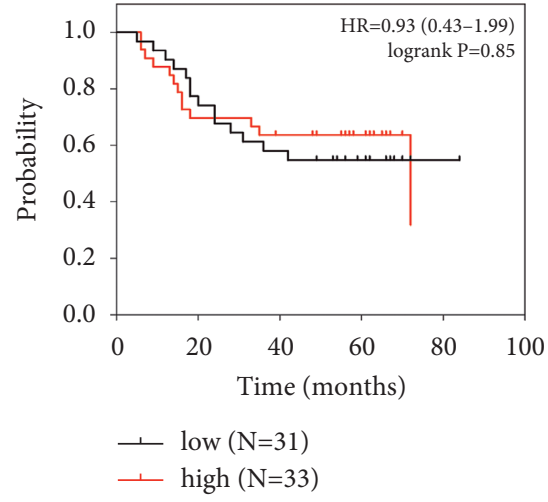

(e)

Figure 5: Kaplan-Meier curves of OS of HCC patients based on GNAO1 expression (low versus high). (a-c) OS analysis of all HCC patients $(n=364)$, stage I-II $(n=253)$, and stage III-IV $(n=87)$ in the Kaplan-Meier plotter database; $(\mathrm{d}-\mathrm{e})$ OS analysis of all HCC patients $(n=79)$ and stage I-II $(n=64)$ based on the IHC results.

demonstrated that early TNM stage and high GNAO1 expression were two independent predictors of late relapse in HCC patients, with GNAO1 expression predicting relapse more accurately. Besides, the AUC of TNM stage performed on HCC relapse was 0.606 in our study, which was similar to the result (0.616) reported by Liu et al. [24]. Furthermore, we successfully constructed a valuable prognostic model by combining GNAO1 expression with TNM stage to more precisely predict the likelihood of relapse in HCC patients. The results demonstrated that the GNAO1 related prognostic model can be used to predict HCC relapse within 3 years, and patients with high GNAO1 expression and early tumor stage have a low relapse rate. $\mathrm{Bu}$ et al. constructed a similar prognostic model to predict survival of patients after colorectal cancer surgery, which has great potential to be used in the real world [25]. 
There were several limitations in the study. We did not analyze the RFS of 15 patients with advanced HCC in the IHC assay. However, it can be concluded from the analysis of the Kaplan-Meier plotter database that advanced patients with high GNAO1 expression also have better RFS. Besides, our HCC tissues were mainly obtained from patients suffering from the HBV $(62 / 79)$, which is the leading cause of HCC in China [26], and the GNAO1 prognostic model was constructed based on retrospective data. Hence, a rich supply of different HCC samples with long-term follow-up information are required to further study the clinical significance of GNAO1, including verifying its prognostic value by recruiting a prospective cohort.

\section{Conclusions}

Our findings provide convincing evidence that GNAO1 is significantly downregulated in HCC tissues and GNAO1 expression can satisfactorily predict HCC relapse. Furthermore, the nomogram consisting of GNAO1 expression and the TNM model presents good ability in predicting the 3 -year relapse, suggesting that it could be a reliable biomarker of relapse prediction as well as a promising therapeutic target for HCC.

\section{Data Availability}

The datasets used in this paper are available from the corresponding author upon request.

\section{Conflicts of Interest}

The authors declare no conflicts of interest.

\section{Authors' Contributions}

Meiling Du and Jie Feng contributed equally to this work.

\section{Acknowledgments}

The authors greatly appreciate the technological help from the department of pathology (Shanghai Fifth People's Hospital, Fudan University, Shanghai, China) for performing the immunohistochemistry assay and results analysis. They also sincerely thank Zhenzhen Zhang (Department of Pathology, Shanghai Ninth People's Hospital, Jiaotong University, Shanghai, China) for her help in pathology. This work was funded by the Natural Science Fund of Minhang District (No. 2019MHZ073).

\section{References}

[1] H. Sung, J. Ferlay, R. L. Siegel et al., "Global cancer statistics 2020: GLOBOCAN estimates of incidence and mortality worldwide for 36 cancers in 185 countries," CA: A Cancer Journal for Clinicians, vol. 71, no. 3, pp. 209-249, 2021.

[2] M. Sayiner, P. Golabi, and Z. M. Younossi, "Disease burden of hepatocellular carcinoma: a global perspective," Digestive Diseases and Sciences, vol. 64, no. 4, pp. 910-917, 2019.

[3] W. Tang, Y. Tu, S. K. Nayak, J. Woodson, M. Jehl, and E. M. Ross, "G $\beta \gamma$ inhibits g $\alpha$ GTPase-activating proteins by inhibition of g $\alpha$-GTP binding during stimulation by receptor," Journal of Biological Chemistry, vol. 281, no. 8, pp. 4746-4753, 2006.

[4] K. Kato, T. Asano, N. Kamiya et al., "Production of the alpha subunit of guanine nucleotide-binding protein GO by neuroendocrine tumors," Cancer Research, vol. 47, no. 21, pp. 5800-5805, 1987.

[5] T. Asano, R. Morishita, and K. Kato, "Identification of a guanine nucleotide-binding protein $\mathrm{G}(\mathrm{o})$ in human neuroblastoma," Cancer Research, vol. 48, no. 10, pp. 2756-2759, 1988.

[6] A. Okumura, K. Maruyama, M. Shibata et al., "A patient with a GNAO1 mutation with decreased spontaneous movements, hypotonia, and dystonic features," Brain and Development, vol. 40, no. 10, pp. 926-930, 2018.

[7] H. Feng, B. Sjögren, B. Karaj, V. Shaw, A. Gezer, and R. R. Neubig, "Movement disorder in GNAO1 encephalopathy associated with gain-of-function mutations," Neurology, vol. 89, no. 8, pp. 762-770, 2017.

[8] M. J. Malaquias, I. Fineza, L. Loureiro, L. Cardoso, I. Alonso, and M. Magalhães, "GNAO1 mutation presenting as dyskinetic cerebral palsy," Neurological Sciences, vol. 40, no. 10, pp. 2213-2216, 2019.

[9] C. M. Honey, A. K. Malhotra, M. Tarailo-Graovac, C. D. M. van Karnebeek, G. Horvath, and A. Sulistyanto, "GNAO1 mutation-induced pediatric dystonic storm rescue with pallidal deep brain stimulation," Journal of Child Neurology, vol. 33, no. 6, pp. 413-416, 2018.

[10] Z. Liu, J. Zhang, L. Wu, J. Liu, and M. Zhang, "Overexpression of GNAO1 correlates with poor prognosis in patients with gastric cancer and plays a role in gastric cancer cell proliferation and apoptosis," International Journal of Molecular Medicine, vol. 33, no. 3, pp. 589-596, 2014.

[11] Y. Yang, F.-H. Chu, W.-R. Xu et al., "Identification of regulatory role of DNA methylation in colon cancer gene expression via systematic bioinformatics analysis," Medicine, vol. 96, no. 47, Article ID e8487, 2017.

[12] N. Hauptman, D. Jevšinek Skok, E. Spasovska, E. Boštjančič, and D. Glavač, "Genes CEP55, FOXD3, FOXF2, GNAO1, GRIA4, and KCNA5 as potential diagnostic biomarkers in colorectal cancer," BMC Medical Genomics, vol. 12, no. 1, p. 54, 2019.

[13] M. Garcia-Marcos, P. Ghosh, and M. G. Farquhar, "Molecular basis of a novel oncogenic mutation in GNAO1," Oncogene, vol. 30, no. 23, pp. 2691-2696, 2011.

[14] X. Pei, J. Zhang, L. Wu et al., "The down-regulation of GNAO1 and its promoting role in hepatocellular carcinoma," Bioscience Reports, vol. 33, no. 5, Article ID e00069, 2013.

[15] D. Xu, M. Du, J. Zhang et al., "DNMT1 mediated promoter methylation of GNAO1 in hepatoma carcinoma cells," Gene, vol. 665, pp. 67-73, 2018.

[16] X. Liu, J. Liu, W. Xiao et al., "SIRT1 regulates N 6 -mmh by inducing RANBP2-dependent FTO SUMOylation," Hepatology, vol. 72, no. 6, pp. 2029-2050, 2020.

[17] D. R. Rhodes, J. Yu, K. Shanker et al., "ONCOMINE: a cancer microarray database and integrated data-mining platform," Neoplasia, vol. 6, no. 1, pp. 1-6, 2004.

[18] B. Györffy, A. Lanczky, A. C. Eklund et al., "An online survival analysis tool to rapidly assess the effect of 22,277 genes on breast cancer prognosis using microarray data of 1,809 patients," Breast Cancer Research and Treatment, vol. 123, no. 3 , pp. 725-731, 2010.

[19] J. Ren, G. Niu, X. Wang, T. Song, Z. Hu, and C. Ke, "Overexpression of FNDC1 in gastric cancer and its 
prognostic significance," Journal of Cancer, vol. 9, no. 24, pp. 4586-4595, 2018.

[20] S. Y. Park, "Nomogram: an analogue tool to deliver digital knowledge," The Journal of Thoracic and Cardiovascular Surgery, vol. 155, no. 4, p. 1793, 2018.

[21] J. Choi, C. Jo, and Y. S. Lim, "Tenofovir versus entecavir on recurrence of hepatitis B virus-related hepatocellular carcinoma after surgical resection," Hepatology, vol. 73, no. 2, pp. 661-673, 2021.

[22] T. T. Zhang, X. Q. Zhao, Z. Liu, Z. Y. Mao, and L. Bai, "Factors affecting the recurrence and survival of hepatocellular carcinoma after hepatectomy: a retrospective study of 601 Chinese patients," Clinical and Translational Oncology, vol. 18, no. 8, pp. 831-840, 2016.

[23] A. Alacacioglu, I. Somali, I. Simsek et al., "Epidemiology and survival of hepatocellular carcinoma in Turkey: outcome of multicenter study," Japanese Journal of Clinical Oncology, vol. 38, no. 10, pp. 683-688, 2008.

[24] H. Liu, Y. Yan, R. Chen et al., "Integrated nomogram based on five stage-related genes and TNM stage to predict 1-year recurrence in hepatocellular carcinoma," Cancer Cell International, vol. 20, no. 1, p. 140, 2020.

[25] L. Bu, F. Huang, M. Li et al., "Identification of Vitamin D-related gene signature to predict colorectal cancer prognosis," PeerJ, vol. 9, Article ID e11430, 2021.

[26] M. Tanaka, F. Katayama, H. Kato et al., "Hepatitis B and C virus infection and hepatocellular carcinoma in China: a review of epidemiology and control measures," Journal of Epidemiology, vol. 21, no. 6, pp. 401-416, 2011. 